\title{
GEOMETRIC DIFFEOMORPHISM FINITENESS IN LOW DIMENSIONS AND HOMOTOPY GROUP FINITENESS
}

\author{
WiLDERICH TUSCHMANN
}

August 1999

\begin{abstract}
Our main result asserts that for any given numbers $C$ and $D$ the class of simply connected closed smooth manifolds of dimension $m<7$ which admit a Riemannian metric with sectional curvature bounded in absolute value by $|K| \leq C$ and diameter uniformly bounded from above by $D$ contains only finitely many diffeomorphism types. Thus in these dimensions the lower positive bound on volume in Cheeger's Finiteness Theorem can be replaced by a purely topological condition, simply-connectedness. In dimension 4 instead of simply-connectedness here only non-vanishing of the Euler characteristic has to be required.

As a topological corollary we obtain that for $k+l<7$ there are over a given smooth closed $l$-manifold only finitely many principal $T^{k}$ bundles with simply connected and non-diffeomorphic total spaces.

Furthermore, for any given numbers $C$ and $D$ and any dimension $m$ it is shown that for each $i \in \mathbb{N}$ there are up to isomorphism always only finitely many possibilities for the $i$ th homotopy group of a simply connected closed $m$-manifold which admits a metric with curvature $|K| \leq C$ and diameter $\leq D$.
\end{abstract}

\section{INTRODUCTION}

The main result of this note asserts that in dimensions less than 7 the presence of a metric with given curvature and diameter bounds suffices to restrict the diffeomorphism type of a simply connected closed manifold to finitely many possibilities:

Theorem 1. For any given numbers $C$ and $D$ there is only a finite number of diffeomorphism types of simply connected closed smooth $m$-manifolds, $m<7$, which admit Riemannian metrics with sectional curvature $|K| \leq C$ and diameter $\leq D$.

Note that Theorem 1 does not require a lower positive bound on volume or injectivity radius.

The above result implies by Myer's theorem that given $m<7$ and any $\delta>0$, there is only a finite number of diffeomorphism types of simply connected closed smooth $m$-dimensional manifolds $M$ which admit Riemannian metrics with Ricci curvature Ricc $\geq \delta>0$ and sectional curvature $K \leq 1$.

This explains in particular why 7 is the first dimension where infinite sequences of closed simply connected manifolds of mutually distinct diffeomorphism type and uniformly positively pinched sectional curvature (see [AW], [E]) can appear.

In view of Theorem 1 it is also interesting to note that there are simply connected four-manifolds of fixed homeomorphism type which admit infinitely many distinct smooth structures (for example $\mathbb{C P}^{2} \# 9 \overline{\mathbb{C P}^{2}}$, see $[\mathrm{FM}]$ ). (In dimension 4 Theorem 1 actually holds for closed smooth manifolds with non-zero Euler characteristic, see below.)

In the relevant dimensions Theorem 1 complements and improves other geometric finiteness results. It shows that for dimensions $m<7$ in Cheeger's Finiteness Theorem the assumption of a lower positive bound on volume can be replaced by a topological condition, namely, simply-connectedness. Theorem 1 also shows that for these dimensions in the $\pi_{2}$-Finiteness Theorem from [PT] the requirement that the second homotopy group be finite is actually not needed. 
The finiteness theorems in Riemannian geometry which require at most bounds on volume, curvature, and diameter can be stated as follows: For manifolds $M$ of a given dimension $m$, the conditions

- $\operatorname{vol}(M) \geq v>0,|K(M)| \leq C$ and $\operatorname{diam}(M) \leq D$ imply finiteness of diffeomorphism types (Cheeger $([\mathrm{C}])$ and Peters $([\mathrm{Pt}]))$; this conclusion moreover continues to hold under the conditions $\operatorname{vol}(M) \geq v>0, \int_{M}|R|^{m / 2} \leq C,\left|\operatorname{Ricc}_{M}\right| \leq C^{\prime}, \operatorname{diam}(M) \leq D$ (Anderson and Cheeger $\left.([\mathrm{AC}])\right)$

- $\operatorname{vol}(M) \geq v>0, K(M) \geq C \operatorname{diam}(M) \leq D$ imply finiteness of homotopy types (Grove and Petersen $([\mathrm{GP}]))$, homeomorphism types (Perelman $([\mathrm{Pr}])$ and Lipschitz homeomorphism types (Perelman, unpublished); if in addition $m \geq 4$, these conditions imply finiteness of homeomorphism, and, if $m>4$, finiteness of diffeomorphism types (Grove, Petersen and Wu ([GPW] ));

- $\pi_{2}(M)$ finite, $\pi_{1}(M)=0,|K(M)| \leq C$ and $\operatorname{diam}(M) \leq D$ imply finiteness of diffeomorphism types $([\mathrm{PT}])$;

- $K(M) \geq C$ and $\operatorname{diam}(M) \leq D$ imply a uniform bound for the total Betti number (Gromov ([G]).

Note that in every dimension $m \geq 7$ there are counterexamples to Theorem 1:

Uniformly positively pinched sequences of mutually non-diffeomorphic Aloff-Wallach or Eschenburg spaces (see $[\mathrm{AW}],[\mathrm{E}]$ ) and their products with spheres of appropiate dimension show that the theorem does not hold in dimension 7 , nor in any dimension $m \geq 9$.

To obtain counterexamples to the validity of Theorem 1 in dimension 8 one can proceed as follows:

Starting with the connected sum $M:=S^{3} \times \mathbb{C P}^{2} \# S^{3} \times \mathbb{C P}^{2}$, using the Gysin sequence one sees that for any pair of relatively prime integers $p, q \in \mathbb{Z}$ there is a circle bundle $S^{1} \rightarrow E_{p, q} \rightarrow M$ over $M$ whose total space is simply connected and whose fourth cohomology is isomorphic to $\mathbb{Z}_{p} \times \mathbb{Z}_{q}$. In particular infinitely many non-diffeomorphic total spaces arise. It only remains to observe that given any Riemannian metric on $M$, one can easily construct on each total space $E_{p, q}$ a metric with similar curvature and diameter bounds as the metric on $M$.

Question. When can the upper curvature bound in Theorem 1 be discarded ?

In dimension 3 the answer might well depend on the still unresolved Poincaré Conjecture.

Combining Gromov's Betti number theorem and Freedman's classification of simply connected topological four-manifolds (see [FQ]) one can see that Theorem 1 holds in dimension 4 under the conditions $K \geq C$ and diam $\leq D$, provided that one replaces diffeomorphism by homeomorphism types. (The reader might wish to compare this with the finiteness theorem of Grove-Petersen-Wu).

In dimension 5 the answer to the above question seems to be positive. Using Barden's diffeomorphism classification of simply connected five-manifolds $([\mathrm{B}])$ and again the Betti number theorem, it can at least be shown that in dimension 5 the conclusion of Theorem 1 holds under the conditions $K(M) \geq C, \operatorname{diam}(M) \leq D$, and $\mid$ Tor $H_{2}(M ; \mathbb{Z}) \mid \leq C^{\prime}$

In the six-dimensional case the upper curvature bound is absolutely necessary for Theorem 1 to hold. This follows from the fact that Karsten Grove and Wolfgang Ziller constructed nonnegatively curved metrics on an infinite sequence of non-diffeomorphic $S^{2}$ bundles over $S^{4}$ (see [GZ]).

The proof of Theorem 1 uses in dimensions 5 and 6 the classification results of Barden ([B]) and Žubr $([\mathrm{Z}])$. Its geometric ingredients consists in collapsing arguments and the following homotopy group finiteness theorem which, in contrast to Theorem 1, is valid in any dimension:

Theorem 2. Given $m \in \mathbb{N}, C$ and $D$, for each natural number $i \geq 2$ there exists a finite set $\Pi_{i}=\Pi_{i}(m, C, D)$ of isomorphism classes of finitely generated Abelian groups that satisfy:

If $M$ is a simply connected closed smooth $m$-dimensional manifold which admits a Riemannian metric with sectional curvature $|K(M)| \leq C$ and diameter $\operatorname{diam}(M) \leq D$, then $\pi_{i}(M) \in \Pi_{i}$.

Theorem 2 follows from a geometric classification theorem for simply connected closed manifolds of $[\mathrm{PT}]$ (see below) and improves Theorem 0.3 in $[\mathrm{R}]$ from finiteness of possibilities for the rational homotopy groups $\pi_{i}(M) \otimes \mathbb{Q}$ to finiteness of possibilities for the homotopy groups $\pi_{i}(M)$ themselves.

I would like to mention that Fang and Rong announced an independent proof of Theorem 1, and it is my pleasure to thank Anton Petrunin and Matthias Kreck, without whom I also would never have found the reference $[\mathrm{H}]$, for help with topology. 


\section{Proofs}

Proof of Theorem 1. In dimension one and two the theorem is true by trivial reasons. Since a simply connected closed three-manifold is a homotopy three-sphere, in dimension three the $\pi_{2^{-}}$ Finiteness Theorem of [PT] applies. Thus we are left with the cases where the dimension $m$ is equal to 4,5 , or 6 .

First note that in a given dimension $m$ Theorem 1 is true for all closed smooth $m$-manifolds with non-zero Euler characteristic. This can be seen as follows:

Suppose that there exists a sequence $\left(M_{n}\right)_{n \in \mathbb{N}}$ of pairwise non-diffeomorphic closed smooth Riemannian $m$-manifolds with uniformly bounded curvatures and diameters and non-zero Euler characteristic. Cheeger's Finiteness Theorem implies that this sequence must collapse, i.e., it must hold that $\operatorname{vol}\left(M_{n}\right) \rightarrow 0$ as $n \rightarrow \infty$. In particular, by [CG] for $n$ sufficiently large all $M_{n}$ admit a pure $F$-structure of positive rank and thus have vanishing Euler characteristic, which is a contradiction.

Since the Euler characteristic of a closed 4-manifold with finite fundamental group is at least 2, Theorem 1 therefore holds in particular for closed smooth 4-manifolds with arbitrarily large, but finite order of the fundamental group.

In the five-dimensional case Barden's classification ([B]) says that simply connected 5-manifolds $M$ are classified up to diffeomorphism by the second homology group $H_{2}(M ; \mathbb{Z})$ and an invariant $i(M)$ which is obtained as follows: Regarding the second Stiefel-Whitney class of $M$ as a homomorphism $w: H_{2}(M) \rightarrow \mathbb{Z}_{2}$, one may arrange $w$ to be non-zero on at most one element of a certain "basis" of $H_{2}$. This element has order $2^{i}$ for some $i$, and this $i$ is the invariant $i(M)$.

Since there are for a given finitely generated group $H$ always only finitely many homomorphisms to a fixed finite group, it follows in particular that there are always only finitely many distinct diffeomorphism types of closed smooth simply connected 5-manifolds with a given second homology group. This observation, combined with Theorem 2 (and the Hurewicz theorem) proves Theorem 1 for $m=5$.

To prove Theorem 1 in dimension six, let us first note that Žubr's diffeomorphism classification of closed oriented simply connected six-manifolds ([Z]) implies that a given class of closed smooth simply connected manifolds of dimension 6 will contain at most finitely many diffeomorphism types if it satisfies the following conditions:

For the members $M$ of this class there are, up to isomophism, only finitely many possibilities for the second homology group $H_{2}(M ; \mathbb{Z})$, the third Betti number $b_{3}(M ; \mathbb{Z})$, the cup form $\mu_{M}$ (a symmetric trilinear form $H^{2}(M ; \mathbb{Z}) \otimes H^{2}(M ; \mathbb{Z}) \otimes H^{2}(M ; \mathbb{Z}) \rightarrow H^{6}(M ; \mathbb{Z}) \cong \mathbb{Z}$ given by the cup product evaluated on the orientation class, which determines the multiplicative structure of the cohomology ring), the first Pontryagin class $p_{1}(M)$ (which here is integral, i.e., $p_{1}(M) \in H^{4}(M ; \mathbb{Z})$ ).

Now suppose that for some $C$ and $D$ there exists an infinite sequence of pairwise non-diffeomorphic closed smooth simply connected Riemannian 6-manifolds $M_{n}$ with curvature $|K| \leq C$ and diameter $\leq D$. By what has been said above, this sequence must collapse, and we may suppose that all manifolds $M_{n}$ carry a pure $F$-structure of positive rank and have vanishing Euler characteristic.

Since $M_{n}$ is simply connected, by [CG] (compare [PT], [R]) the $F$-structure on each $M_{n}$ is given by an effective smooth torus action without fixed points. Since all orbits of this action have positive dimension, it is easy to see that each $M_{n}$ thus also admits a fixed-point free circle action.

Now ([H], Lemma 3.2) implies that each $M_{n}$ has vanishing trilinear cup form $\mu$ and that the first Pontryagin class of $M_{n}$ is torsion, i.e., $p_{1}\left(M_{n}\right) \in$ Tor $H^{4}\left(M_{n} ; \mathbb{Z}\right)$. By Poincaré duality, Tor $H^{4}\left(M_{n} ; \mathbb{Z}\right) \cong$ Tor $H_{2}\left(M_{n} ; \mathbb{Z}\right)$. Also note that $\chi\left(M_{n}\right)=0$ implies that $b_{3}\left(M_{n}\right)=2 b_{2}\left(M_{n}\right)+2$.

But by Theorem 2 there are only finitely many possibilities for the second homology group of all $M_{n}$. Combining this with the above facts it follows that our collapsing sequence contains at most finitely non-diffeomorphic manifolds, which yields the desired contradiction.

Since the total space of a principal torus bundle over a Riemannian manifold always carries a metric with similar curvature and diameter bounds as the base, Theorem 1 has the following

Topological Corollary. For $k+l<7$ there are over a given smooth closed $l$-manifold only finitely many principal $T^{k}$ bundles with simply connected and non-diffeomorphic total spaces. 
Theorem 2 is a direct corollary of the following classification theorem for simply connected closed manifolds from [PT]:

Theorem ([PT]). For given $m \in \mathbb{N}, C$ and $D$, there exists a finite number of closed smooth manifolds $E_{l}$ such that any simply connected closed $m$-dimensional manifold $M$ admitting a Riemannian metric with sectional curvature $|K| \leq C$ and diameter $\leq D$ is diffeomorphic to a factor space $M=E_{l} / T^{k_{l}}$, where $0 \leq k_{l}=\operatorname{dim} E_{l}-m$ and $T^{k_{l}}$ acts freely on $E_{l}$.

Proof of Theorem 2. Fix numbers $m, C$, and $D$, and let $M$ be a closed smooth simply connected of dimension $m$ which admits a Riemannian metric with sectional curvature $|K| \leq C$ and diameter $\leq D$. By the above theorem there exists a closed smooth simply connected manifold $E$ such that $E$ is diffeomorphic to the total space of a principal $T^{k}$ bundle over $M$. (In the terminology of [PT], the manifold $E$ is the universal torus bundle of $M$, where $\pi_{2}(E)$ is finite and $0 \leq k=b_{2}(M)$.

Since tori are aspherical, the homotopy exact sequence

$$
\cdots \rightarrow \pi_{i}\left(T^{k}\right) \rightarrow \pi_{i}(E) \rightarrow \pi_{i}(M) \rightarrow \cdots \rightarrow 0=\pi_{2}\left(T^{k}\right) \rightarrow \pi_{2}(E) \rightarrow \pi_{2}(M) \rightarrow \pi_{1}\left(T^{k}\right) \cong \mathbb{Z}^{k} \rightarrow 0
$$

of the principal bundle $T^{k} \rightarrow E \rightarrow M$ then shows that for $3 \leq i \in \mathbb{N}$ the homotopy group $\pi_{i}(M)$ is isomorphic to $\pi_{i}(E)$, and that $\pi_{2}(M)$ is isomorphic to $\pi_{2}(E) \oplus \mathbb{Z}^{k}$.

Since by the above theorem for given numbers $m, C$, and $D$ there are only finitely many nondiffeomorphic manifolds $E$, in noting that the homotopy groups of a closed simply connected manifold are finitely generated one sees that the proof of Theorem 2 is complete.

\section{REFERENCES}

[AC] M. Anderson, J. Cheeger, Diffeomorphism finiteness for manifolds with Ricci curvature and $L^{n / 2}$-norm of curvature bounded, GAFA (Geometric and functional analysis) 1 (1991), 231-252.

[AW] S. Aloff; N. R. Wallach, An infinite family of 7-manifolds admitting positively curved Riemannian structures, Bull. Amer. Math. Soc. 81 (1975), 93-97.

[B] D. Barden, Simply-connected 5-manifolds, Ann. of Math. 68 (1958), 721-734.

[C] J. Cheeger, Finiteness theorems for Riemannian manifolds, Amer. J. Math. 92 (1970), 61-74.

[CG] J. Cheeger; M. Gromov, Collapsing Riemannian manifolds while keeping their curvature bound I, II, J. Diff. Geom. 23, 32 (1986, 1990), 309-364 and 269-298.

[E] J.-H. Eschenburg, New examples of manifolds with strictly positive curvature, Invent. math. 66 (1982), 469480.

[FM] R. Friedman; J.W. Morgan, On the diffeomorphism type of certain algebraic surfaces I, J. Diff. Geom 27 (1988), 297-369.

[FQ] M. Freedman; F. Quinn, Topology of 4-manifolds, Princeton Univ. Press, Princeton, New Jersey (1990).

[G] M. Gromov, Curvature, diameter and Betti numbers, Comment. Math. Helv. 56 (1981), 179-195.

[GP] K. Grove; P. Petersen, Bounding homotopy types by geometry, Ann. of Math. 128 (1988), 195-206.

[GPW] K. Grove; P. Petersen; J. Wu, Controlled topology in geometry, Invent. Math. 99 (1990), 205-213; Erratum: Invent. Math. 104 (1991), 221-222.

[GZ] K. Grove; W. Ziller, Curvature and symmetry of Milnor spheres. http://www.math.upenn.edu/ wziller/papers groveziller.

$[\mathrm{H}] \quad$ W. Huck, A note on circle actions on 5-and 6-dimensional manifolds, Konstanzer Schriften in Mathematik und Informatik Nr. 27 (1997), http://www.informatik.uni-konstanz.de/Schriften.

[Pr] G. Perelman, Alexandrov Spaces with curvature bounded below II, Preprint.

[Pt] S. Peters, Cheeger's finiteness theorem for diffeomorphism classes of Riemannian manifolds, J. Reine Angew. Math 349, 77-82.

[PT] A. Petrunin; W. Tuschmann, Diffeomorphism Finiteness, Positive Pinching, and Second Homotopy, GAFA (Geometric and functional analysis), to appear.

[R] X. Rong, Bounding homotopy and homology groups by curvature and diameter, Duke Math. J 78,2 (1995), 427-435.

[Z] A.V. Žubr, Classification of simply-connected topological 6-manifolds, Topology and Geometry (RohlinSeminar), Springer Lecture Notes in Mathematics 1346 (1988), 325-339.

Max-Planck-Institute for Mathematics in the Sciences, Inselstrasse, D-04103 Leipzig, Germany

E-mail address: tusch@mis.mpg.de 